ARTICLE

https://doi.org/10.1038/s41467-019-09047-7

\title{
Phase segregation due to ion migration in all-inorganic mixed-halide perovskite nanocrystals
}

Huichao Zhang ${ }^{1,2}$, Xu Fu', Ying Tang ${ }^{1}$, Hua Wang ${ }^{3}$, Chunfeng Zhang ${ }^{1}$, William W. Yu (D) ${ }^{3}$, Xiaoyong Wang ${ }^{1}$, Yu Zhang ${ }^{3} \&$ Min Xiao ${ }^{1,4}$

Semiconductor mixed-halide perovskites featured with a tunable energy bandgap are ideal candidates for light absorbers in tandem solar cells as well as fluorescent materials in lightemitting diodes and nanoscale lasers. These device advancements are currently hindered by the light-induced phase segregation effect, whereby ion migration would yield smaller-bandgap domains with red-shifted photoluminescence. Here we show that upon laser excitation allinorganic mixed-halide nanocrystals unexpectedly exhibit a blue shift in the photoluminescence peak that can revert back in the dark, thus depicting the processes of ion migration out of and back to the originally excited nanocrystals. Interestingly, this reversible photoluminescence shift can also be induced by electrical biasing of mixed-halide nanocrystals without the injection of charge carriers. The above findings suggest that it is the local electric field that breaks the ionic bonds in mixed-halide nanocrystals, which could be a universal origin for light-induced phase segregation observed in other mixed-halide perovskite materials.

\footnotetext{
${ }^{1}$ National Laboratory of Solid State Microstructures, School of Physics, and Collaborative Innovation Center of Advanced Microstructures, Nanjing University, Nanjing 210093, China. ${ }^{2}$ College of Electronics and Information, Hangzhou Dianzi University, Xiasha Campus, Hangzhou 310018, China. ${ }^{3}$ State Key Laboratory of Integrated Optoelectronics and College of Electronic Science and Engineering, Jilin University, Changchun 130012, China. ${ }^{4}$ Department of Physics, University of Arkansas, Fayetteville, AR 72701, USA. These authors contributed equally: Huichao Zhang, Xu Fu. Correspondence and requests for materials should be addressed to X.W. (email: wxiaoyong@nju.edu.cn) or to Y.Z. (email: yuzhang@jlu.edu.cn) or to M.X. (email: mxiao@uark.edu)
} 
S emiconductor perovskites of methylammonium (MA) lead halide $\left(\mathrm{MAPbX}_{3}, \mathrm{X}=\mathrm{Br}, \mathrm{Cl}\right.$, I or mixtures thereof) have been drawing great research attention due to their superior performance in solar cells with a rapidly rising photo-conversion efficiency toward that of the crystalline silicon counterparts ${ }^{1,2}$. The main obstacle for commercial advancement of these organolead halide solar cells lies in the instability and degradation of light-harvesting $\mathrm{MAPbX}_{3}$ films ${ }^{3}$. Especially bothersome is the ion migration effect, which is believed to be responsible for the slow conductivity response ${ }^{4}$, anomalous current-voltage hysteresis ${ }^{5,6}$, giant dielectric constant ${ }^{7}$, and switchable photocurrent ${ }^{8}$ in the $\mathrm{MAPbI}_{3}$ perovskites. Meanwhile, ion migration is manifested in mixed-halide $\mathrm{MAPbBr}_{x} \mathrm{I}_{3-x}(0<x<3)$ perovskites as a lightinduced segregation of iodine- and bromine-rich domains that can revert back to the original phase in the dark ${ }^{9-12}$. The iodinerich domains in the $\mathrm{MAPbBr}_{x} \mathrm{I}_{3-x}$ perovskites would cause a red shift in the photoluminescence (PL) that has been similarly observed in the $\mathrm{CsPbBr}_{x} \mathrm{I}_{3-x}$ perovskites ${ }^{13-16}$, confirming a universal role played by the migration of halide ions in the lightinduced phase segregation process. In addition to the bulk mixedhalide perovskites mentioned above, the phase segregation effect has also been observed in the $\mathrm{CsPbBr}_{x} \mathrm{I}_{3-x}$ nanocrystals (NCs) albeit from the electroluminescence measurements ${ }^{17,18}$. Given the nanoscale size of the iodine-rich domains ${ }^{11,19}$ that are preferentially located along grain boundaries of the bulk mixedhalide perovskites ${ }^{11,16,20,21}$, the $\mathrm{CsPbBr}_{x} \mathrm{I}_{3-x}$ NCs with a normally defective surface could provide a succinct understanding of the phase segregation process from a bottom-up point of view.

Here we focus on mixed-halide $\mathrm{CsPbBr}{ }_{1.2} \mathrm{I}_{1.8} \mathrm{NCs}$ to investigate the phase segregation effect at both the ensemble-film and the single-particle levels. With laser excitation, the ensemble film of CsPbBr ${ }_{1.2} \mathrm{I}_{1.8}$ NCs demonstrates a blue shift from about 630 to $520 \mathrm{~nm}$ in the PL peak that can revert back in the dark, which can be attributed to the migration of iodide ions out of and back to the originally excited NCs. For an isolated single $\mathrm{CsPbBr}_{1.2} \mathrm{I}_{1.8}$ $\mathrm{NC}$, the PL is also shifted to the blue side upon laser excitation but never returns back in the dark, signifying the necessary existence of nearby NCs to channel the migration of iodide ions. Interestingly, the blue-shifted PL can also be induced when the $\mathrm{CsPbBr}{ }_{1.2} \mathrm{I}_{1.8}$ NCs are electrically biased in the dark without the injection of excited-state charge carriers. This strongly suggests that it is the local electric field to break the iodide bonds that triggers the ion migration process in photo-excited $\mathrm{CsPbBr}_{1.2} \mathrm{I}_{1.8}$ NCs. While the enlarged energy bandgap is mainly caused by bromine enrichment in mixed-halide $\mathrm{CsPbr}_{1.2} \mathrm{I}_{1.8} \mathrm{NCs}$, we additionally show that lattice distortion by the migration of iodide ions could also make a minor contribution, as verified by the observation of a blue shift in the PL peak as large as $20 \mathrm{~nm}$ from the single-halide $\mathrm{CsPb}_{3} \mathrm{NCs}$ upon laser excitation.

\section{Results}

Chemical synthesis and experimental conditions. According to a previously reported procedure ${ }^{22}$ (see Methods), mixedhalide $\mathrm{CsPbBr}{ }_{1.2} \mathrm{I}_{1.8}$ NCs were similarly synthesized with a cubic shape and a side length of about $23 \mathrm{~nm}$ as estimated from the transmission electron microscopy (TEM) measurements (Supplementary Figure 1). From room-temperature solution measurements, the absorption and emission peaks of the $\operatorname{CsPbBr}_{1.2} \mathrm{I}_{1.8}$ $\mathrm{NCs}$ are located at 617 and $632 \mathrm{~nm}$, respectively (Supplementary Figure 2). One drop of the concentrated or diluted solution of the $\mathrm{CsPbBr}{ }_{1.2} \mathrm{I}_{1.8}$ NCs was spin-coated onto a fused silica substrate for the room-temperature optical characterizations of an ensemble film or single particles with a $405 \mathrm{~nm}$ picosecond laser operated at a repetition rate of $5 \mathrm{MHz}$ (see Methods). The laser beam was focused onto the sample substrate with a spot size of about 500 $\mathrm{nm}$ so that different positions of the ensemble film or the single particles could be studied with a high spatial resolution.

Optical measurements of high-density $\mathrm{CsPbBr}_{1.2} \mathrm{I}_{1.8}$ NCs. As shown in Fig. 1a for one position of the ensemble film of $\mathrm{CsPbBr}{ }_{1.2} \mathrm{I}_{1.8}$ NCs, the PL peak was blue-shifted from the initial $635 \mathrm{~nm}$ toward a stable value of $618 \mathrm{~nm}$ after $10 \mathrm{~min}$ of continuous laser excitation at the power density of $30 \mathrm{~W} \mathrm{~cm}^{-2}$ (in comparison, the power density of 1 sun illumination is $100 \mathrm{~mW}$ $\mathrm{cm}^{-2}$ ). The reversibility of the above process is demonstrated in Fig. $1 \mathrm{~b}$, where the same position of the ensemble film showed a red shift in the PL peak that approached $631 \mathrm{~nm}$ after $15 \mathrm{~min}$ of being left in the dark. The above PL shifting process is strongly dependent on the laser excitation power, as can be seen in Fig. 1c for another position of the ensemble film excited at $15 \mathrm{~kW} \mathrm{~cm}^{-2}$. Within 80 min of laser excitation, the PL peak was blue-shifted all the way toward about $520 \mathrm{~nm}$ to coincide with optical emission from the single-halide $\mathrm{CsPbBr}_{3} \mathrm{NCs}^{18,23}$, while similar blueshifted behavior was also observed in the corresponding absorption spectrum (Supplementary Figure 3). When this position of the ensemble film was then measured in the dark (Fig. 1d), the PL peak was also red-shifted but stopped only at $553 \mathrm{~nm}$ after 120 min to demonstrate a worse reversibility than that obtained at $30 \mathrm{~W} \mathrm{~cm}^{-2}$. Accompanying time-dependent shift of the PL peak, PL intensity of the ensemble film was also partially reversible, dropping by about $45 \%$ (90\%) immediately after laser excitation at $30 \mathrm{~W} \mathrm{~cm}^{-2}\left(15 \mathrm{~kW} \mathrm{~cm}^{-2}\right)$ and recovering in the dark to about $65 \%(45 \%)$ of the original value.

For the bulk films of mixed-halide perovskites ${ }^{10,12}$, lightinduced phase segregation is universally causing a red shift in the PL due to exciton trapping into the iodine-rich regions with a smaller energy bandgap than that of the bromine-rich regions $9,15,24$. However, laser excitation here on the ensemble film of $\mathrm{CsPbBr}_{1.2} \mathrm{I}_{1.8}$ NCs reproducibly yielded a blue shift in the $\mathrm{PL}$, suggesting that they were being transformed to the brominerich optical emitters owing to the preferred bond breaking and the subsequent escape of iodide ions. This can be largely understood since it has been documented both theoretically and experimentally that the $\mathrm{Pb}-\mathrm{Br}$ bond is shorter and stronger than the $\mathrm{Pb}-\mathrm{I}$ bond ${ }^{24-29}$, so that a higher binding energy would be associated with the ionization of the bromide ions ${ }^{30}$. In the bulk films of mixed-halide $\mathrm{MAPbBr}_{x} \mathrm{I}_{3-x}$ and $\mathrm{CsPbBr}_{x} \mathrm{I}_{3-x}$ perovskites, the light-induced iodine-rich domains are normally located at the grain boundaries $11,16,20,21$ that are full of impurities, vacancies or dangling bonds ${ }^{31}$. These grain boundaries can be well mimicked by the defective surfaces of $\mathrm{CsPbBr}{ }_{1.2} \mathrm{I}_{1.8} \mathrm{NCs}$, and they can be envisioned to serve as a stopping site into which the light-freed iodide ions can diffuse.

Optical measurements of low-density and single $\mathrm{CsPbBr}_{1.2} \mathrm{I}_{1.8}$ NCs. We next diluted the sample solution by 1000 times to make a low-density ensemble film of $\mathrm{CsPbBr}_{1.2} \mathrm{I}_{1.8}$ NCs, whose PL peak was blue-shifted to $512 \mathrm{~nm}$ after $20 \mathrm{~min}$ of laser excitation at 120 $\mathrm{W} \mathrm{cm}^{-2}$ and stayed there almost permanently with only a slight recovery to the original position in the dark (Supplementary Figure 4). We further diluted the above sample solution to make a solid film containing spatially isolated single $\mathrm{CsPbBr}_{1.2} \mathrm{I}_{1.8} \mathrm{NCs}$, which could be verified from the second-order photon correlation measurement (Supplementary Figure 5). As shown in Fig. 2a for a representative single $\mathrm{CsPbBr}_{1.2} \mathrm{I}_{1.8} \mathrm{NC}$ excited at $6 \mathrm{~W} \mathrm{~cm}^{-2}$, the initial $615 \mathrm{~nm}$ PL peak was blue-shifted to 570 and $515 \mathrm{~nm}$ after 25 and $50 \mathrm{~min}$ of continuous laser excitation, which never reverted back to the red side in the dark. This irreversible blue shift in the PL was observed in all of the single $\mathrm{CsPbBr}_{1.2} \mathrm{I}_{1.8}$ NCs studied in our experiment and, in some cases, a slight blue shift 

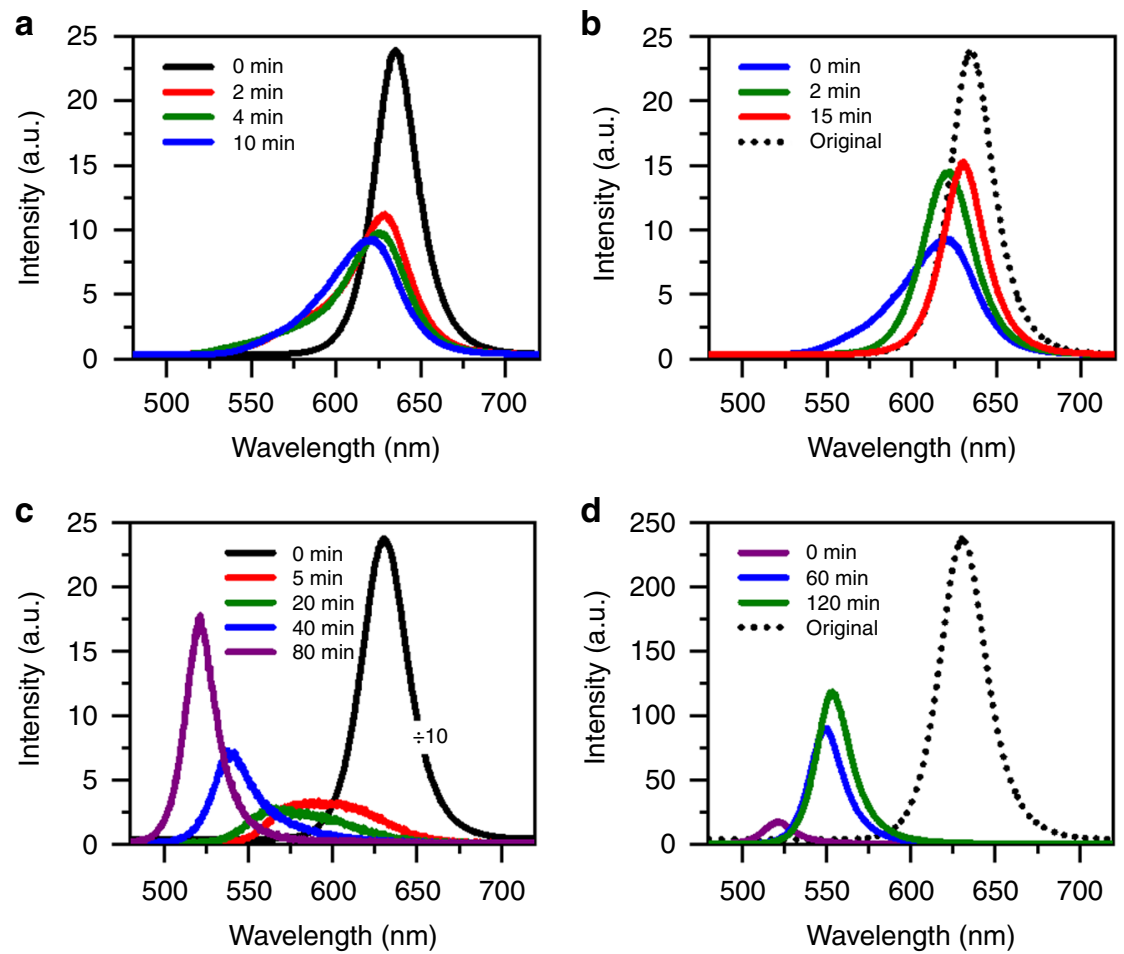

Fig. 1 Light-induced phase segregation in ensemble film of $\mathrm{CsPbr}_{1.2} \mathrm{l}_{1.8}$ nanocrystals (NCs). a Photoluminescence (PL) spectra measured at 0,2 , 4, and $10 \mathrm{~min}$ for one position of the ensemble film continuously excited at a laser power density of $30 \mathrm{~W} \mathrm{~cm}^{-2}$. The PL spectrum at each time point was acquired with an integration time of $1 \mathrm{~s}$. b PL spectra measured for the same position of the ensemble film after the excitation laser had then been blocked for 0 , 2 , and $15 \mathrm{~min}$. At each time point, the laser beam was unblocked for $1 \mathrm{~s}$ to acquire the PL spectrum still at $30 \mathrm{~W} \mathrm{~cm}^{-2}$. The original PL spectrum measured at the beginning of the laser excitation in a is also plotted. c PL spectra measured at 0, 5, 20, 40, and 80 min for another position of the ensemble film continuously excited at a laser power density of $15 \mathrm{~kW} \mathrm{~cm}-2$. The PL spectrum at each time point was acquired with an integration time of $1 \mathrm{~s}$. The PL spectrum measured at 0 min has been scaled down by 10 times for a good comparison. $\mathbf{d}$ PL spectra measured for this position of the ensemble film after the excitation laser had then been blocked for 0,60, and $120 \mathrm{~min}$. At each of the above time points, the laser beam was unblocked for $1 \mathrm{~s}$ to acquire the PL spectrum still at $15 \mathrm{~kW} \mathrm{~cm}^{-2}$. The original PL spectrum measured at the beginning of the laser excitation in $\mathbf{c}$ is also plotted

could be further resolved in the dark. As can be seen in Fig. $2 \mathrm{~b}$ for a single $\mathrm{CsPbBr}_{1.2} \mathrm{I}_{1.8} \mathrm{NC}$ excited at $6 \mathrm{Wcm}^{-2}$, the PL peak was blue-shifted from 616 to $513 \mathrm{~nm}$ after 4 min of laser excitation and moved to $508 \mathrm{~nm}$ after the excitation laser beam had been blocked for $20 \mathrm{~min}$ in the dark.

The reversible PL shift could only occur in the high-density ensemble film due to ion migration among nearby $\mathrm{CsPbBr}{ }_{1.2} \mathrm{I}_{1.8}$ NCs, which is reminiscent of the anion-exchange interaction between solution $\mathrm{CsPbBr}_{3}$ and $\mathrm{CsPbI}_{3} \mathrm{NCs}$ to form the mixedhalide $\mathrm{CsPbBr}_{x} \mathrm{I}_{3-x} \mathrm{NCs}^{32}$. As suggested previously for bulk $\mathrm{MAPbI}_{3}$ films ${ }^{31}$, the iodide ions would migrate away from the light-illuminated area to the immediately adjacent regions due to the Coulomb repulsion effect. Once the light illumination is removed, the stored iodide ions in the surroundings could be driven by the concentration gradient ${ }^{31}$ or the mixing entropy ${ }^{21,33}$ to fill the vacancies left in the original position. The above scenario can be similarly applied to the high-density $\mathrm{CsPbBr}_{1.2} \mathrm{I}_{1.8}$ NCs, so long as they can be treated as a bulk film divided into numerous nanoscale domains by the intervening surfaces. The situation is quite different for the low-density ensemble film and the isolated single $\mathrm{CsPbBr}_{1.2} \mathrm{I}_{1.8}$ NCs showing a monotonic and irreversible blue shift in the PL. This can be temporarily attributed to the reduction of surface iodide ions and the subsequent sublimation into a gaseous form ${ }^{34,35}$, which have been shown to be quite efficient in bulk films of the $\mathrm{MAPbI}_{3}$ perovskites especially under the influence of an electric field ${ }^{36}$. Within a high-density film of $\mathrm{CsPbBr}_{1.2} \mathrm{I}_{1.8} \mathrm{NCs}$, the iodide ions would be dominantly channeled away from the laser excitation area, but part of them might still suffer from the above dissipation process in some isolated NCs. This could be a likely origin for the incomplete recoveries of the PL peak and intensity shown in Fig. 1b, $\mathrm{d}$ for the high-density ensemble film of $\mathrm{CsPbBr}{ }_{1.2} \mathrm{I}_{1.8} \mathrm{NCs}$ in the dark. We performed energy-dispersive spectroscopy (EDS) measurements on a high-density film of ensemble $\mathrm{CsPbBr}{ }_{1.2} \mathrm{I}_{1.8}$ NCs, whose PL peak was first blue-shifted to about $520 \mathrm{~nm}$ under high-power laser excitation and then moved back in the dark to a stable value of about $600 \mathrm{~nm}$. EDS characterizations were applied on both laser-unirradiated and -irradiated areas to yield the $\mathrm{I} / \mathrm{Br}$ stoichiometric ratios of about 1.46 and 1.28 , respectively, thus confirming the loss of iodine element in the light-induced degradation process.

In bulk perovskite films, it has been firmly established that trap states on the surface and at the grain boundary can capture photo-excited charge carriers to create a local electric field capable of promoting ion migration $31,37,38$. When an electron-hole pair is photo-excited in a single colloidal $\mathrm{NC}$, one of the charge carriers (e.g., the electron) can be trapped on the surface, leaving behind an extra hole in the core that couples with the next electron-hole pair to form a charged exciton 39,40 . The NC would become nonemissive due to nonradiative Auger recombination of the charged exciton, while the PL intensity can be resumed only after the surface electron returns back to or the extra hole also leaves the core ${ }^{39,40}$. This PL blinking effect was also observed here in single $\mathrm{CsPbBr}{ }_{1.2} \mathrm{I}_{1.8} \mathrm{NCs}$, as can be seen in Fig. $2 \mathrm{c}$ for a representative one whose PL intensity switched randomly between the bright and dark time periods. For a single electron trapped on the CdSe 

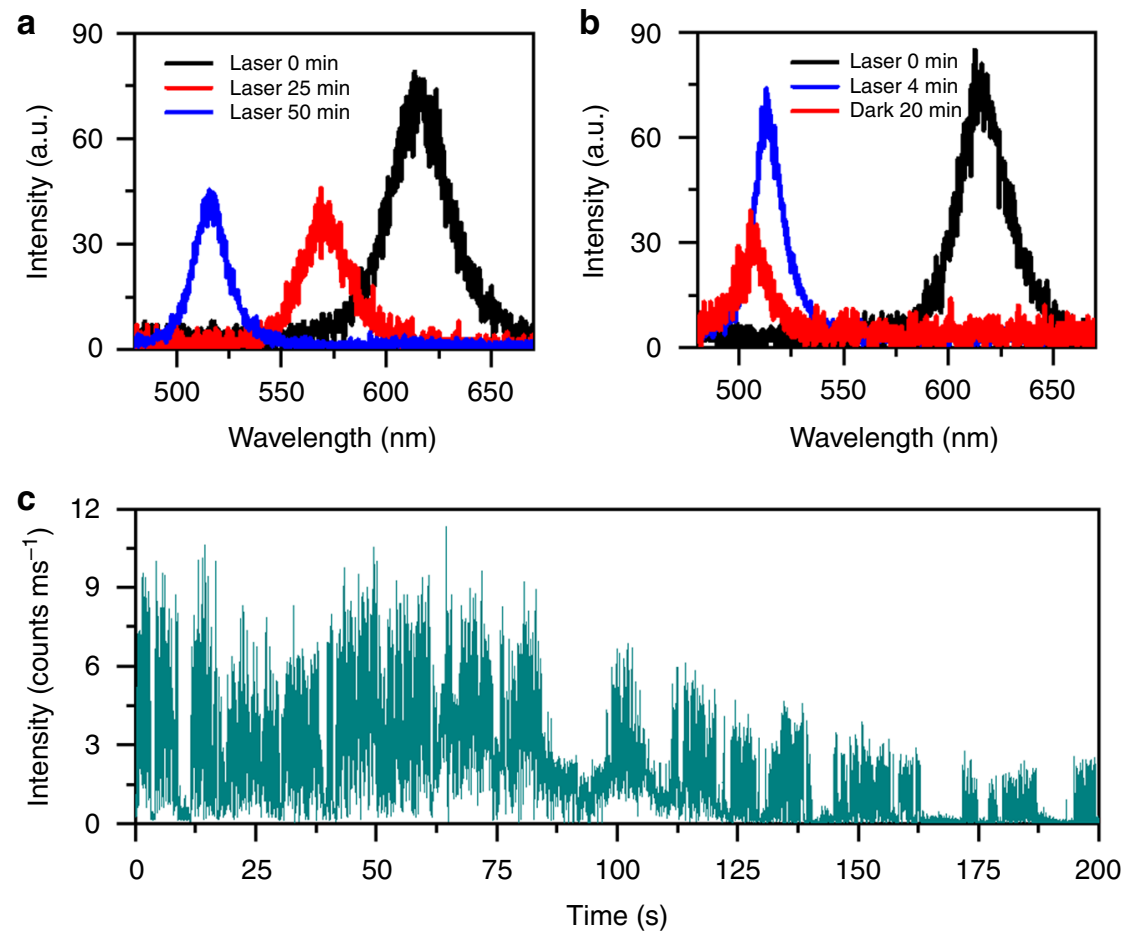

Fig. 2 Light-induced phase segregation in single $\mathrm{CsPbBr}_{1.2} \mathrm{l}_{1.8}$ nanocrystals (NCs). a Photoluminescence (PL) spectra measured at three time points of 0 , 25 , and 50 min with an integration time of $1 \mathrm{~s}$ for a single $\mathrm{CsPbBr}_{1.2} \mathrm{l}_{1.8} \mathrm{NC}$ continuously excited at a laser power density of $6 \mathrm{~W} \mathrm{~cm}{ }^{-2}$. b PL spectra measured at two time points of 0 and 4 min with an integration time of $1 \mathrm{~s}$ for a single $\mathrm{CsPbBr}_{1.2} \mathrm{l}_{1.8} \mathrm{NC}$ continuously excited at a laser power density of $6 \mathrm{~W} \mathrm{~cm}{ }^{-2}$. Then after the excitation laser beam had been blocked for $20 \mathrm{~min}$, a PL spectrum was taken with an integration time of $1 \mathrm{~s}$ for this single NC also excited at $6 \mathrm{~W} \mathrm{~cm}^{-2}$. c Time-dependent PL intensity measured between 20 and 220 s for the single $\mathrm{CsPbBr}_{1.2} \mathrm{l}_{1.8} \mathrm{NC}$ whose PL spectra are plotted in $\mathbf{b}$ and continuously excited at $6 \mathrm{~W} \mathrm{~cm}-2$. The binning time for acquiring each data point for the PL intensity is $100 \mathrm{~ms}$

NC surface, a local electric field from several to tens of volts per micrometer could be created ${ }^{41}$. It should be this electric field that breaks iodide bonds in $\mathrm{CsPbBr}{ }_{1.2} \mathrm{I}_{1.8} \mathrm{NCs}$, possibly by creating a local strain in an inverse piezoelectric process, which has been theoretically proposed in molecular dynamics simulations on the bulk films of $\mathrm{MAPbBr}_{x} \mathrm{I}_{3-x}$ perovskites ${ }^{11}$. Recently, a compressive strain of $1 \%$ was reported for the bulk films of $\mathrm{MAPbI}_{3}$ and $\mathrm{CsPbI}_{3}$ perovskites biased under an electric field of $3.7 \mathrm{~V} \mathrm{\mu m^{-1 }}$ (ref. ${ }^{42}$ ), thus lending support to the above proposal. It should be noted that the high-power laser excitation employed in our experiment could create multiple excitons in a single $\mathrm{CsPbBr}{ }_{1.2} \mathrm{I}_{1.8}$ $\mathrm{NC}$ to trigger the exciton-exciton annihilation effect ${ }^{43}$. This kind of Auger-mediated process would greatly increase the charging possibility of a single $\mathrm{NC}^{40}$ to make it more vulnerable to the local electric field, which could explain the larger blue shift observed in the PL peak of ensemble $\mathrm{CsPbBr}_{1.2} \mathrm{I}_{1.8}$ NCs under higher-power laser excitation (Fig. 1a, c).

Electrical biasing of high-density $\mathrm{CsPbBr}_{1.2} \mathrm{I}_{1.8}$ NCs. To verify the influence of electric field on the phase segregation effect, we placed one solution drop of high-density $\mathrm{CsPbBr}_{1.2} \mathrm{I}_{1.8} \quad \mathrm{NCs}$ between two electrodes each with a width of $10 \mu \mathrm{m}$ and separated by $5 \mu \mathrm{m}$ in an interdigitated pattern. From bottom to top, the $\mathrm{Au}$ and $\mathrm{Cr}$ layers were deposited with the thicknesses of 80 and $5 \mathrm{~nm}$, respectively. When an external electric field was applied between the two electrodes, there was no current flowing through the NC film so that electrical injection of charge carriers could be avoided. As shown in Fig. 3a for one position of the NC film, the PL intensity dropped significantly and the PL peak was blue-shifted from 631 to $607 \mathrm{~nm}$ after an electrical biasing of $10 \mathrm{~V}$ had been continuously supplied for $4 \mathrm{~min}$ in the dark. When this external electrical field was removed, the PL intensity recovered to about $20 \%$ of the initial value while the PL peak was red-shifted to reach $619 \mathrm{~nm}$ after the NC film had been left in the dark for $10 \mathrm{~min}$ (see Supplementary Figure 6 for a similar experiment performed on a low-density film of ensemble $\mathrm{CsPbBr}_{1.2} \mathrm{I}_{1.8}$ NCs where no red-shifted PL was observed after the external electric field had been removed). Consequently, the application of an electric field of $2 \mathrm{~V} \mu \mathrm{m}^{-1}$ here to $\mathrm{CsPbBr}{ }_{1.2} \mathrm{I}_{1.8}$ NCs could induce a similar PL shifting behavior to that from the laser excitation (see Fig. 1a, b). This is dramatically different from nearly all of the previous phase segregation studies on mixed-halide perovskites, wherein the excited-state charge carriers were exclusively required from either light illumination ${ }^{10,12}$ or electrical injection ${ }^{17,18}$. It was proposed that these charge carriers could couple with the soft lattice to create a polaron $7,44,45$, while the resulting strain would be sufficient to drive halide segregation for the nucleation of iodine-rich domains ${ }^{11,15,46,47}$. This polaron picture is obviously not applicable to the phase segregation effect observed here in our $\mathrm{CsPbBr}{ }_{1.2} \mathrm{I}_{1.8} \mathrm{NCs}$, the function of whose photo-excited charge carriers should be the creation of a local electric field after being trapped on the surface, as can be deduced from the single-particle PL blinking phenomenon displayed in Fig. 2c.

Besides contributing to polaron formation, the excited-state charge carriers have also been deduced to drive the hopping of halide vacancies ${ }^{48,49}$ in the crystal lattice with an activation energy ranging from about 0.1 to $0.6 \mathrm{eV}$ for mixed-halide perovskites $9,21,24,26,31,37,50$. In this sense, the external electric field applied by us to the high-density $\mathrm{CsPbBr}_{1.2} \mathrm{I}_{1.8} \mathrm{NCs}$ might serve a similar role to energetically migrate iodide ions into their vacancies far away from the detection area. In this vacancy picture for an isolated single $\mathrm{Cs} \mathrm{PbBr}_{1.2} \mathrm{I}_{1.8}$ NC under laser excitation, the final destination for the migrated iodide ions could 

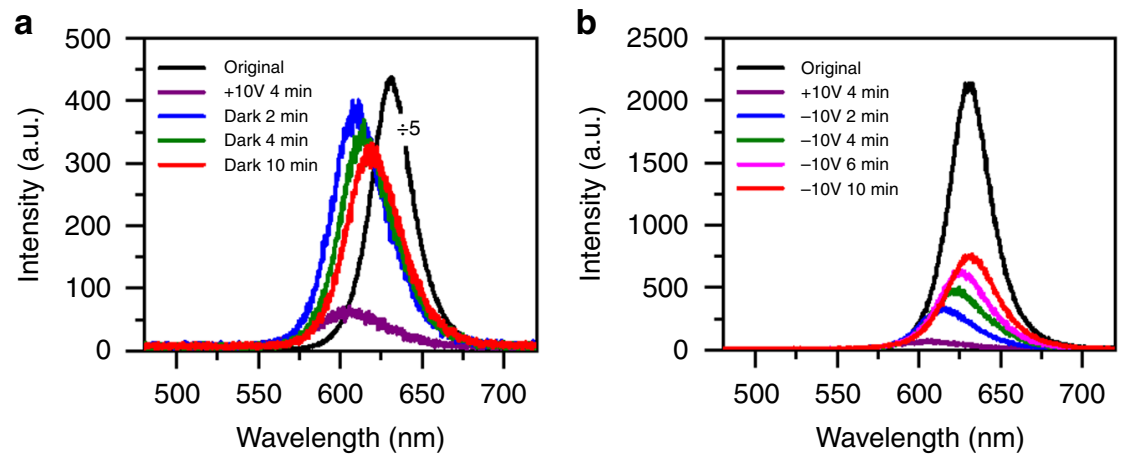

Fig. 3 Phase segregation in ensemble film of $\left.\mathrm{CsPbBr}_{1.2}\right|_{1.8}$ nanocrystals (NCs) under electrical biasing. a Original photoluminescence (PL) spectrum measured for one position of the ensemble film without electrical biasing, together with the PL spectrum of this position measured after the ensemble film had been electrically biased for $4 \mathrm{~min}$ at $10 \mathrm{~V}$ in the dark. Also shown are three PL spectra of this position measured after the electrical biasing had then been removed for 2, 4, and $10 \mathrm{~min}$. b Original PL spectrum measured for one position of the ensemble film without electrical biasing, together with the PL spectrum of this position measured after the ensemble film had been electrically biased for 4 min at $10 \mathrm{~V}$ in the dark. Also shown are four PL spectra of this position measured after a reverse electrical biasing of $10 \mathrm{~V}$ had been continuously supplied in the dark for $2,4,6$, and 10 min. To acquire each of the PL spectra shown in $\mathbf{a}$ and $\mathbf{b}$, the laser beam was unblocked for $1 \mathrm{~s}$ to excite the ensemble film with a power density of $6 \mathrm{~W} \mathrm{~cm}-2$

only be the surface vacancies where their $\mathrm{Pb}$-I bond would be still conserved. It is then impossible for all of these iodide ions to sublime away and some of them should move back in the dark to fill the leftover vacancies in the core, which contradicts a further blue shift in the PL shown in Fig. $2 b$ for a typical single $\mathrm{CsPbBr}{ }_{1.2} \mathrm{I}_{1.8}$ NC. Now neglecting the polaron and vacancy origins for the phase segregation process, we can reasonably assume that the iodide ions should stay loosely on the surface or as interstitials in the $\mathrm{CsPbBr}_{1.2} \mathrm{I}_{1.8}$ NC lattice after the Pb-I bond is broken by the local or external electric field.

In Fig. 3b, we plot the PL spectra measured for one position of the high-density film of $\mathrm{CsPbBr}_{1.2} \mathrm{I}_{1.8}$ NCs formed between two other electrodes, which was also blue-shifted from 631 to $607 \mathrm{~nm}$ under an electrical biasing of $10 \mathrm{~V}$ for $4 \mathrm{~min}$ in the dark. When this electrical biasing was reversed to $-10 \mathrm{~V}$ and maintained for $10 \mathrm{~min}$ in the dark, the $607 \mathrm{~nm}$ PL peak could shift all the way back to the initial $631 \mathrm{~nm}$, while an elongated biasing would shift this PL peak to the blue side again. Compared to natural recovery in the dark for the PL peak shown in Fig. 3a, the application of a reverse electric field obviously accelerated reflowing process of the freed iodide ions. For a specific single $\mathrm{Cs} \mathrm{PbBr}_{1.2} \mathrm{I}_{1.8} \mathrm{NC}$, the number of returning iodide ions should be larger than that of the escaping ones whose $\mathrm{Pb}-\mathrm{I}$ bonds are broken again by the electric field, which can be invoked to explain the continuous red shift as well as the complete recovery of the PL peak observed in Fig. $3 b$.

Other mechanisms for blue-shifted PL. Besides the migration of iodide ions to yield bromine-rich perovskite NCs, it is necessary to figure out whether there exist other mechanisms that can cause an enlarged energy bandgap with the blue-shifted PL. It is well known that the energy bandgap of all-inorganic perovskite NCs would increase with the increasing temperature ${ }^{23}$, however, this laser heating possibility can be safely ruled out since the blueshifted PL was also induced by the application of an electric field (Fig. 3a). Meanwhile, the cooling process of $\mathrm{CsPbBr}_{1.2} \mathrm{I}_{1.8}$ NCs should not be different in the dark for a high-density ensemble film and an isolated single NC, but no returning of the PL to the red side was observed in the latter case (Fig. 2a, b). Moreover, we also excited ensemble $\operatorname{CsPbBr}_{1.2} \mathrm{I}_{1.8}$ NCs with a laser energy below their bandgap without seeing any blue-shifted PL (Supplementary Figure 7), thus again excluding possible contribution of the laser heating effect. Another possible mechanism for the variation of energy bandgap could be lattice distortion in $\mathrm{CsPbBr}_{1.2} \mathrm{I}_{1.8}$ NCs after the migration of iodide ions ${ }^{12}$. It was shown previously in bulk $\mathrm{MAPbI}_{3}$ perovskites ${ }^{51-53}$ that light illumination could alter the $\mathrm{Pb}$-I bond angle and length, causing either lattice expansion or contraction that was able to decrease or increase the energy bandgap, respectively. To completely exclude the contribution of bromine enrichment to the enlarged energy bandgap, we synthesized single-halide perovskite $\mathrm{CsPbI}_{3} \mathrm{NCs}$ (see Methods) to probe whether the migration of iodide ions and the subsequent lattice distortion could occur to induce a blue shift in the PL.

Compared to mixed-halide $\mathrm{CsPbBr}_{1.2} \mathrm{I}_{1.8}$ NCs whose lightinduced phase segregation could be initiated at a laser power density smaller than $30 \mathrm{~W} \mathrm{~cm}^{-2}$ (Fig. 1a), the single-halide

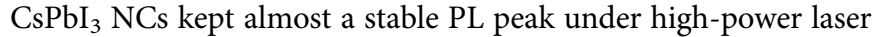
excitation up to $10 \mathrm{~kW} \mathrm{~cm}^{-2}$. As shown in Fig. 4a for one position of the high-density film of ensemble $\mathrm{CsPbI}_{3} \mathrm{NCs}$, the PL peak was blue-shifted from the initial 689 to $678 \mathrm{~nm}$ after $2.5 \mathrm{~min}$ of laser excitation at $15 \mathrm{~kW} \mathrm{~cm}^{-2}$. It then shifted back in the dark to arrive at $688 \mathrm{~nm}$ after $40 \mathrm{~min}$, with the original intensity being completely recovered (Fig. 4b). The blue shift observed here in the $\mathrm{PL}$ peak of ensemble $\mathrm{CsPbI}_{3} \mathrm{NCs}$, which could revert back to the red side in the dark, is different from that shown previously in single $\mathrm{CsPbI}_{3} \mathrm{NCs}$ where layer-by-layer decomposition caused a permanent shrinkage of the $\mathrm{NC}$ size $\mathrm{s}^{54}$. With elongated laser excitation still at $15 \mathrm{~kW} \mathrm{~cm}^{-2}$, the PL peak monitored for another position on the $\mathrm{CsPbI}_{3} \mathrm{NC}$ film was blue-shifted from 688 to $668 \mathrm{~nm}$ after $10 \mathrm{~min}$ with a drastic intensity drop by about 100 times (Fig. 4c). When the NC film was left in the dark, the PL peak of the same position shifted to the red side and stabilized at $675 \mathrm{~nm}$ after $150 \mathrm{~min}$, while the PL intensity recovered partially to about $30 \%$ of the original value (Fig. $4 \mathrm{~d}$ ). We further diluted the sample solution by 15 times to make a low-density film of ensemble $\mathrm{CsPbI}_{3} \mathrm{NCs}$, whose PL peak measured for one position was blue-shifted from 689 to $682 \mathrm{~nm}$ after $10 \mathrm{~min}$ of laser excitation at $15 \mathrm{~kW} \mathrm{~cm}^{-2}$ (Supplementary Figure 8). This PL peak still stayed at $682 \mathrm{~nm}$ even after the ensemble NC film had been left in the dark for $150 \mathrm{~min}$, implying that the migration of iodide ions did occur among the high-density $\mathrm{CsPbI}_{3} \mathrm{NCs}$ according to our previous discussions on the mixed-halide CsPbBr ${ }_{1.2} \mathrm{I}_{1.8}$ NCs (see Fig. 2a, b, as well as Supplementary Figure 4). The above optical measurements on the single-halide $\mathrm{CsPbI}_{3} \mathrm{NCs}$ strongly suggest that, in addition to the dominant role played by bromine enrichment, a minor contribution of 

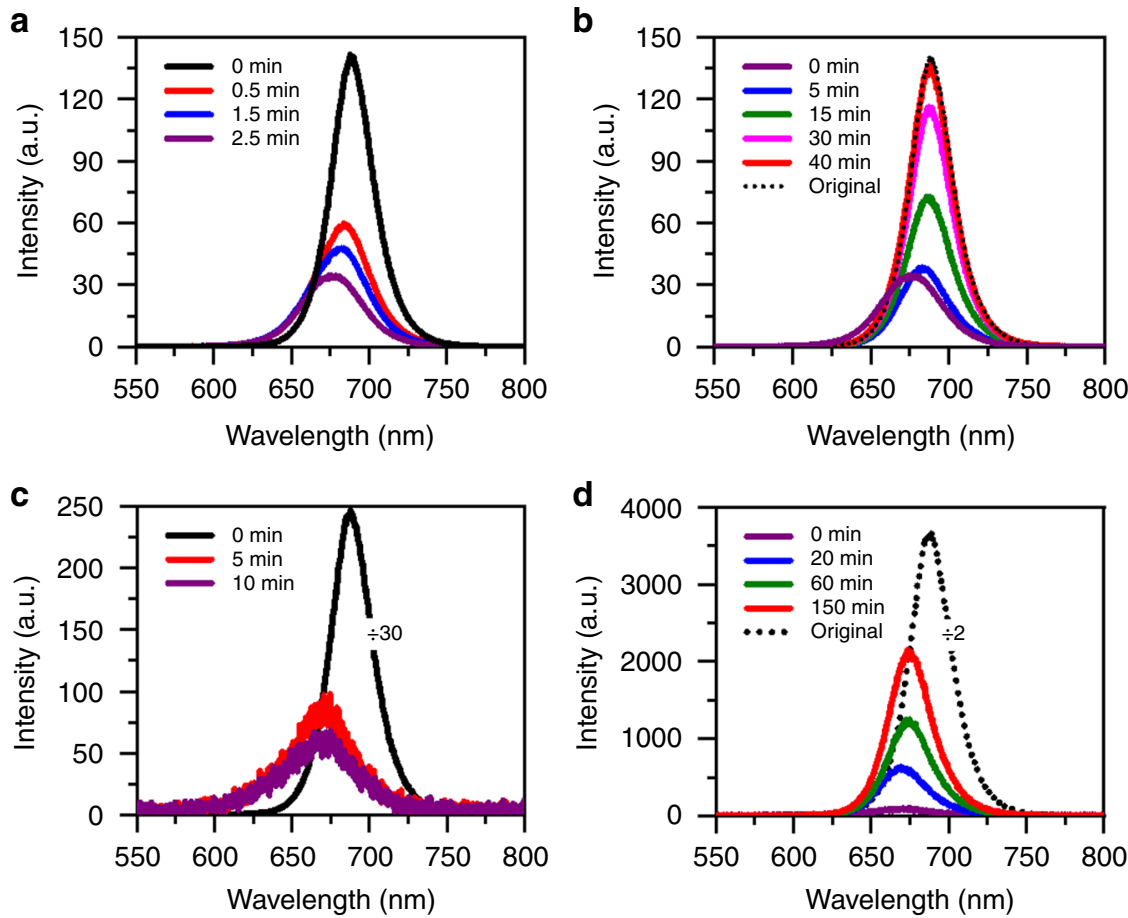

Fig. 4 Light-induced migration of iodide ions in ensemble film of $\mathrm{CsPb}_{3}$ nanocrystals (NCs). a Photoluminescence (PL) spectra measured at 0 , 0.5 , 1.5, and 2.5 min for one position of the ensemble film continuously excited at a laser power density of $15 \mathrm{~kW} \mathrm{~cm}^{-2}$. The PL spectrum at each time point was acquired with an integration time of $1 \mathrm{~s}$. b PL spectra measured for the same position of the ensemble film after the excitation laser had then been blocked for $0,5,15,30$, and $40 \mathrm{~min}$. At each time point, the laser beam was unblocked for $1 \mathrm{~s}$ to acquire the PL spectrum still at $15 \mathrm{~kW} \mathrm{~cm}^{-2}$. The original PL spectrum measured at the beginning of the laser excitation in a is also plotted. c PL spectra measured at 0,5 , and 10 min for another position of the ensemble film continuously excited at a laser power density of $15 \mathrm{~kW} \mathrm{~cm}^{-2}$. The PL spectrum at each time point was acquired with an integration time of $1 \mathrm{~s}$. The PL spectrum measured at 0 min has been scaled down by 30 times for a good comparison. d PL spectra measured for this position of the ensemble film after the excitation laser had then been blocked for $0,20,60$, and 150 min. At each time point, the laser beam was unblocked for $1 \mathrm{~s}$ to acquire the PL spectrum still at $15 \mathrm{~kW} \mathrm{~cm}^{-2}$. The original PL spectrum measured at the beginning of the laser excitation in $\mathbf{c}$ is also plotted but scaled down by two times

lattice distortion by the migration of iodide ion to the enlarged energy bandgap should not be neglected in the phase segregation process of $\mathrm{CsPbBr} \mathrm{P}_{1.2} \mathrm{I}_{1.8}$ NCs.

\section{Discussion}

In conclusion, the phase-segregation effect is manifested in mixed-halide $\mathrm{CsPbBr} \mathrm{Pb}_{1.2} \mathrm{I}_{1.8}$ NCs as a blue shift in the PL peak upon laser excitation, which can return back to the red side in the dark for the high-density ensemble film but not the isolated single NCs. Since this blue-shifted PL can also be induced by electrical biasing of the ensemble film, we believe that the light-induced phase segregation process should be triggered by a local electric field generated after the photo-excited charge carriers are trapped on the NC surface. This local electric field would exert strain on the crystal lattice to cause bond breaking of the iodide ions, which then migrate away from the laser-excitation area in the high-density ensemble film or sublime into a gaseous form from the surface of isolated single $\mathrm{CsPbBr}{ }_{1.2} \mathrm{I}_{1.8}$ NCs. Except the blueshifted PL, we believe that the above discussions could also be employed to describe the phase segregation process observed in all the other mixed-halide perovskite materials.

It is well known that light-induced phase segregation in the bulk film of mixed-halide perovskites is universally causing a red shift in the PL peak due to the formation of both bromine- and iodine-rich domains, with the photo-excited excitons being trapped in the latter one with a smaller energy bandgap. The formation of both bromine- and iodine-rich domains in a single
$\mathrm{CsPbBr}{ }_{1.2} \mathrm{I}_{1.8} \mathrm{NC}$ could be safely ruled out since a light-induced blue shift was observed in the PL peak. Meanwhile, recovery of this PL peak to the red side could be observed in the dark only for high-density films of ensemble $\mathrm{CsPbBr}_{1.2} \mathrm{I}_{1.8} \mathrm{NCs}$, thus strongly suggesting that the iodide ions do migrate among neighboring NCs. Since a spatial size of $\sim 8 \mathrm{~nm}^{11}$ is normally needed for the formation of iodine-rich domain in the bulk films of mixedhalide perovskites, we speculate that this requirement might be difficult to be fulfilled in a single $\mathrm{CsPbBr}{ }_{1.2} \mathrm{I}_{1.8}$ NC studied here with a lateral size of $\sim 23 \mathrm{~nm}$.

\section{Methods}

Cs-oleate precursor preparation. The Cs-oleate precursor was prepared by loading a mixture of $0.814 \mathrm{~g} \mathrm{Cs}_{2} \mathrm{CO}_{3}, 2.5 \mathrm{~mL}$ oleic acid $(\mathrm{OA})$ and $30 \mathrm{~mL}$ octadecene (ODE) into a $100 \mathrm{~mL}$ three-neck flask. Under vacuum, the solution was dried and degassed for $10 \mathrm{~min}$, and then heated at $120^{\circ} \mathrm{C}$ for $1 \mathrm{~h}$. The resulting solution was further heated to $150^{\circ} \mathrm{C}$ under $\mathrm{N}_{2}$ gas and kept for $2 \mathrm{~h}$ until a clear solution was obtained. After being stored at room temperature, the Cs-oleate solution was always preheated to $120^{\circ} \mathrm{C}$ before being used for the syntheses of $\mathrm{CsPbBr}{ }_{1.2} \mathrm{I}_{1.8}$ and $\mathrm{CsPbI}_{3} \mathrm{NCs}$.

Synthesis of mixed-halide CsPbBr $\mathbf{P b}_{\mathbf{1 . 2}} \mathbf{l}_{\mathbf{1 . 8}}$ NCs. $0.105 \mathrm{~g} \mathrm{PbI}_{2}, 0.052 \mathrm{~g} \mathrm{PbBr}, 1.0 \mathrm{~mL}$ $\mathrm{OA}, 1.0 \mathrm{~mL}$ oleylamine $(\mathrm{OAm})$ and $10 \mathrm{~mL}$ ODE were loaded into a $25 \mathrm{~mL}$ 3-neck flask. Under vacuum, the mixture solution in the flask was degassed for $10 \mathrm{~min}$ and then dried for $1 \mathrm{~h}$ at $120^{\circ} \mathrm{C}$. After the flask was connected to $\mathrm{N}_{2}$ gas, the solution temperature was raised to $160^{\circ} \mathrm{C}$ and kept for $10 \mathrm{~min}$, after which $1.0 \mathrm{~mL}$ of the Cs-oleate solution preheated to $120^{\circ} \mathrm{C}$ was quickly injected and the reaction was stopped with ice bath after $5 \mathrm{~s}$. The formed $\mathrm{CsPbBr}_{1.2} \mathrm{I}_{1.8} \mathrm{NCs}$ were precipitated by adding tert-butanol and centrifuged for $20 \mathrm{~min}$ at $5000 \mathrm{rpm}$. The precipitate was redispersed in $2.0 \mathrm{~mL}$ hexane and centrifuged again for 
$20 \mathrm{~min}$ at $5000 \mathrm{rpm}$. Finally, the products were dispersed in $10 \mathrm{~mL}$ hexane or toluene and centrifuged for $10 \mathrm{~min}$ at $5000 \mathrm{rpm}$ to remove any possible aggregates.

\section{Synthesis of single-halide $\mathbf{C s P b}_{\mathbf{3}} \mathbf{N C s} 0.174 \mathrm{~g} \mathrm{PbI}_{2}, 1.0 \mathrm{~mL} \mathrm{OA}, 1.0 \mathrm{~mL} \mathrm{OAm}$} and $10 \mathrm{~mL}$ ODE were mixed in a $25-\mathrm{mL}$ three-neck flask. After being degassed for $10 \mathrm{~min}$, the mixture was heated to $120^{\circ} \mathrm{C}$ and kept for $1 \mathrm{~h}$ under vacuum. The flask was then connected to $\mathrm{N}_{2}$ gas and the solution temperature was raised to $160^{\circ} \mathrm{C}$. $1.0 \mathrm{~mL}$ of the Cs-oleate solution preheated to $120^{\circ} \mathrm{C}$ was quickly injected into the hot flask and the reaction was stopped with ice bath after $5 \mathrm{~s}$. The resulting solution was centrifuged for $20 \mathrm{~min}$ at $5000 \mathrm{rpm}$, while the precipitated $\mathrm{CsPbI}_{3} \mathrm{NCs}$ were dispersed in $2.0 \mathrm{~mL}$ hexane and centrifuged again for $20 \mathrm{~min}$ at $5000 \mathrm{rpm}$ for further purification. Finally, the products were dispersed in $10 \mathrm{~mL}$ hexane or toluene and centrifuged for $10 \mathrm{~min}$ at $5000 \mathrm{rpm}$ to remove any possible aggregates.

Optical characterizations. The $\mathrm{CsPbBr}{ }_{1.2} \mathrm{I}_{1.8}$ and $\mathrm{CsPbI}_{3} \mathrm{NC}$ samples could be used directly after synthesis or stored for several months in a glove box filled with Ar gas, while very similar phase-segregation properties were observed from the fresh and stored samples. One drop of the concentrated or diluted solution of colloidal $\mathrm{NCs}\left(\mathrm{CsPbBr}{ }_{1.2} \mathrm{I}_{1.8}\right.$ or $\left.\mathrm{CsPbI}_{3}\right)$ was spin-coated onto a fused silica substrate to form a solid film for the ensemble or single-particle optical characterizations at room temperature. The $405 \mathrm{~nm}$ output of a picosecond diode laser operated at a repetition rate of $5 \mathrm{MHz}$ was focused onto the sample substrate by an immersion-oil objective (numerical aperture, 1.4). PL signal of the ensemble NCs or a single NC was collected by the same objective and sent through a $0.5 \mathrm{~m}$ spectrometer to a chargecoupled-device camera for the PL spectral measurement with an integration time of $1 \mathrm{~s}$. The PL signal of a single NC can be alternatively sent through a non-polarizing 50/50 beam splitter to two avalanche photodiodes for the second-order photon autocorrelation measurement. When excited at the laser power densities specified in the text, the $\mathrm{CsPbBr}_{1.2} \mathrm{I}_{1.8}$ and $\mathrm{CsPbI}_{3} \mathrm{NCs}$ always showed a decrease in the PL intensity and a blue shift in the PL peak at the beginning of the optical measurement, and no PL enhancement was observed from the photo-activation effect previously reported for semiconductor perovskite materials ${ }^{55}$

\section{Data availability}

The data supporting the findings of this study are available from the corresponding authors upon request.

Received: 15 November 2018 Accepted: 18 February 2019

Published online: 06 March 2019

\section{References}

1. Kojima, A., Teshima, K., Shirai, Y. \& Miyasaka, T. Organometal halide perovskites as visible-light sensitizers for photovoltaic cells. J. Am. Chem. Soc. 131, 6050-6051 (2009)

2. National Renewable Energy Labs Efficiency Chart (2018) https://www.nrel. gov/pv/assets/images/efficiency-chart.png (accessed 31 August 2018).

3. Hodes, G. \& Cahen, D. Photovoltaics: perovskite cells roll forward. Nat. Photon 8, 87-88 (2014).

4. Gottesman, R. et al. Extremely slow photoconductivity response of $\mathrm{CH}_{3} \mathrm{NH}_{3} \mathrm{PbI}_{3}$ perovskites suggesting structural changes under working conditions. J. Phys. Chem. Lett. 5, 2662-2669 (2014).

5. Unger, E. L. et al. Hysteresis and transient behavior in current-voltage measurements of hybrid-perovskite absorber solar cells. Energy Environ. Sci. 7, 3690-3698 (2014).

6. Tress, W. et al. Understanding the rate-dependent J-V hysteresis, slow time component, and aging in $\mathrm{CH}_{3} \mathrm{NH}_{3} \mathrm{PbI}_{3}$ perovskite solar cells: the role of a compensated electric field. Energy Environ. Sci. 8, 995-1004 (2015)

7. Juarez-Perez, E. J. et al. Photoinduced giant dielectric constant in lead halide perovskite solar cells. J. Phys. Chem. Lett. 5, 2390-2394 (2014).

8. Xiao, Z. et al. Giant switchable photovoltaic effect in organometal trihalide perovskite devices. Nat. Mater. 14, 193-198 (2015).

9. Hoke, E. T. et al. Reversible photo-induced trap formation in mixed-halide hybrid perovskites for photovoltaics. Chem. Sci. 6, 613-617 (2015).

10. Slotcavage, D. J., Karunadasa, H. I. \& McGehee, M. D. Light-induced phase segregation in halide-perovskite absorbers. ACS Energy Lett. 1, 1199-1205 (2016)

11. Bischak, C. G. et al. Origin of reversible photoinduced phase separation in hybrid perovskites. Nano. Lett. 17, 1028-1033 (2017)

12. Brennan, M. C., Draguta, S., Kamat, P. V. \& Kuno, M. Light-induced anion phase segregation in mixed halide perovskites. ACS Energy Lett. 3, 204-213 (2018).

13. Braly, I. L. et al. Current induced phase segregation in mixed halide hybrid perovskites and its impact on two-terminal tandem solar cell design. ACS Energy Lett. 2, 1841-1847 (2017).
14. Beal, R. E. et al. Cesium lead halide perovskites with improved stability for tandem solar cells. J. Phys. Chem. Lett. 7, 746-751 (2016).

15. Draguta, S. et al. Rationalizing the light-induced phase separation of mixed halide organic-inorganic perovskites. Nat. Commun. 8, 200 (2017).

16. $\mathrm{Li}, \mathrm{W}$. et al. Phase segregation enhanced ion movement in efficient inorganic CsPbIBr 2 solar cells. Adv. Energy Mater. 7, 1700946 (2017).

17. Li, G. et al. Highly efficient perovskite nanocrystal light-emitting diodes enabled by a universal crosslinking method. Adv. Mater. 28, 3528-3534 (2016).

18. Vashishtha, P. \& Halpert, J. E. Field-driven ion migration and color instability in red-emitting mixed-halide perovskite nanocrystal light-emitting diodes. Chem. Mater. 29, 5965-5973 (2017).

19. Gratia, P. et al. Intrinsic halide segregation at nanometer scale determines the high efficiency of mixed cation/mixed halide perovskite solar cells. J. Am. Chem. Soc. 138, 15821-15824 (2016).

20. Rehman, W. et al. Photovoltaic mixed-cation lead mixed-halide perovskites: links between crystallinity, photo-stability and electronic properties. Energy Environ. Sci. 10, 361-369 (2017).

21. Tang, $X$. et al. Local observation of phase segregation in mixed-halide perovskite. Nano. Lett. 18, 2172-2178 (2018).

22. Wang, H. et al. Emission recovery and stability enhancement of inorganic perovskite quantum dots. J. Phys. Chem. Lett. 9, 4166-4173 (2018).

23. $\mathrm{Hu}, \mathrm{F}$. et al. Superior optical properties of perovskite nanocrystals as single photon emitters. ACS Nano 9, 12410-12416 (2015).

24. Yoon, S. J. et al. Tracking iodide and bromide ion segregation in mixed halide lead perovskites during photoirradiation. ACS Energy Lett. 1, 290-296 (2016).

25. Benavides-Garcia, M. \& Balasubramanian, K. Bond energies, ionization potentials, and the singlet-triplet energy separations of $\mathrm{SnCl}_{2}, \mathrm{SnBr}_{2}, \mathrm{SnI}_{2}$ $\mathrm{PbCl}_{2}, \mathrm{PbBr}_{2}, \mathrm{PbI}_{2}$, and their positive ions. J. Chem. Phys. 100, 2821-2830 (1994).

26. Haruyama, J., Sodeyama, K., Han, L. \& Tateyama, Y. First-principles study of ion diffusion in perovskite solar cell sensitizers. J. Am. Chem. Soc. 137, 10048-10051 (2015).

27. Misra, R. K. et al. Temperature- and component-dependent degradation of perovskite photovoltaic materials under concentrated sunlight. J. Phys. Chem. Lett. 6, 326-330 (2015)

28. Yoon, S. J., Stamplecoskie, K. G. \& Kamat, P. V. How lead halide complex chemistry dictates the composition of mixed halide perovskites. J. Phys. Chem Lett. 7, 1368-1373 (2016)

29. Ruess, R., Benfer, F., Böcher, F., Stumpp, M. \& Schlettwein, D. Stabilization of organic-inorganic perovskite layers by partial substitution of iodide by bromide in methylammonium lead iodide. ChemPhysChem 17, 1505-1511 (2016).

30. Lindblad, R. et al. Electronic structure of $\mathrm{CH}_{3} \mathrm{NH}_{3} \mathrm{PbX}_{3}$ perovskites: dependence on the halide moiety. J. Phys. Chem. C 119, 1818-1825 (2015).

31. deQuilettes, D. W. et al. Photo-induced halide redistribution in organicinorganic perovskite films. Nat. Commun. 7, 11683 (2016).

32. Nedelcu, G. et al. Fast anion-exchange in highly luminescent nanocrystals of cesium lead halide perovskites $\left(\mathrm{CsPbX}_{3}, \mathrm{X}=\mathrm{Cl}, \mathrm{Br}, \mathrm{I}\right)$. Nano. Lett. 15, 5635-5640 (2015)

33. Barker, A. J. et al. Defect-assisted photoinduced halide segregation in mixedhalide perovskite thin films. ACS Energy Lett. 2, 1416-1424 (2017).

34. Li, Y. et al. Light-induced degradation of $\mathrm{CH}_{3} \mathrm{NH}_{3} \mathrm{PbI}_{3}$ hybrid perovskite thin film. J. Phys. Chem. C 121, 3904-3910 (2017).

35. Huang, W., Yoon, S. J. \& Sapkota, P. Effect of light illumination on mixed halide lead perovskites: reversible or irreversible transformation. ACS Appl. Energy Mater. 1, 2859-2865 (2018)

36. Zhao, Y.-C. et al. Quantification of light-enhanced ionic transport in lead iodide perovskite thin films and its solar cell applications. Light Sci. Appl. 6 , e16243 (2017)

37. Azpiroz, J. M., Mosconi, E., Bisquert, J. \& De Angelis, F. Defect migration in methylammonium lead iodide and its role in perovskite solar cell operation. Energy Environ. Sci. 8, 2118-2127 (2015).

38. Ahn, N. et al. Trapped charge-driven degradation of perovskite solar cells Nat. Commun. 7, 13422 (2016)

39. Nirmal, M. et al. Fluorescence intermittency in single cadmium selenide nanocrystals. Nature 383, 802-804 (1996).

40. $\mathrm{Hu}, \mathrm{F}$. et al. Slow Auger recombination of charged excitons in nonblinking perovskite nanocrystals without spectral diffusion. Nano. Lett. 16, 6425-6430 (2016).

41. Empedocles, S. A. \& Bawendi, M. G. Quantum-confined stark effect in single CdSe nanocrystallite quantum dots. Science 278, 2114-2117 (1997).

42. Chen, B. et al. Large electrostrictive response in lead halide perovskites. Nat. Mater. 17, 1020-1026 (2018)

43. Makarov, N. S. et al. Spectral and dynamical properties of single excitons, biexcitons, and trions in cesium-lead-halide perovskite quantum dots. Nano. Lett. 16, 2349-2362 (2016). 
44. Zhu, X.-Y. \& Podzorov, V. Charge carriers in hybrid organic-inorganic lead halide perovskites might be protected as large polarons. J. Phys. Chem. Lett. 6, 4758-4761 (2015).

45. Nie, W. et al. Light-activated photocurrent degradation and self-healing in perovskite solar cells. Nat. Commun. 7, 11574 (2016).

46. Hoffman, J. B., Schleper, A. L. \& Kamat, P. V. Transformation of sintered $\mathrm{CsPbBr}_{3}$ nanocrystals to cubic $\mathrm{CsPbI}_{3}$ and gradient $\mathrm{CsPbBr}_{x} \mathrm{I}_{3-x}$ through halide exchange. J. Am. Chem. Soc. 138, 8603-8611 (2016).

47. Bischak, C. G. et al. Tunable polaron distortions control the extent of halide demixing in lead halide perovskites. J. Phys. Chem. Lett. 9, 3998-4005 (2018).

48. Yoon, S. J., Kuno, M. \& Kamat, P. V. Shift happens. How halide ion defects influence photoinduced segregation in mixed halide perovskites. ACS Energy Lett. 2, 1507-1514 (2017).

49. Ruth, A. et al. Vacancy-mediated anion photosegregation kinetics in mixed halide hybrid perovskites: coupled kinetic Monte Carlo and optical measurements. ACS Energy Lett. 3, 2321-2328 (2018).

50. Eames, C. et al. Ionic transport in hybrid lead iodide perovskite solar cells. Nat. Commun. 6, 7497 (2015).

51. Merdasa, A. et al. Super-resolution luminescence microspectroscopy reveals the mechanism of photoinduced degradation in $\mathrm{CH}_{3} \mathrm{NH}_{3} \mathrm{PbI}_{3}$ perovskite nanocrystals. J. Phys. Chem. C 120, 10711-10719 (2016).

52. Tsai, H. et al. Light-induced lattice expansion leads to high-efficiency perovskite solar cells. Science 360, 67-70 (2018).

53. Boyer-Richard, S. et al. Symmetry-based tight binding modeling of halide perovskite semiconductors. J. Phys. Chem. Lett. 7, 3833-3840 (2016).

54. Yuan, G. et al. The degradation and blinking of single $\mathrm{CsPbI}_{3}$ perovskite quantum dots. J. Phys. Chem. C 122, 13407-13415 (2018).

55. Tian, Y. et al. Mechanistic insights into perovskite photoluminescence enhancement: light curing with oxygen can boost yield thousandfold. Phys. Chem. Chem. Phys. 17, 24978-24987 (2015).

\section{Acknowledgements}

This work is supported by the National Key R\&D Program of China (2017YFA0303700), the National Natural Science Foundation of China (11574147, 11621091, 61605037,

51772123, 61675086, and 61722504), China Postdoctoral Science Foundation

(2016M591820), and the PAPD of Jiangsu Higher Education Institutions.

\section{Author contributions}

X.W., C.Z., and M.X. conceived and designed the experiments. W.Y., H.W., and Y.Z. prepared the samples. H.Z., X.F., and Y.T. performed the optical and electrical experiments. X.W. and H.Z. analyzed the data. X.W., C.Z., Y.Z., and M.X. co-wrote the manuscript.

\section{Additional information}

Supplementary Information accompanies this paper at https://doi.org/10.1038/s41467019-09047-7.

Competing interests: The authors declare no competing interests.

Reprints and permission information is available online at http://npg.nature.com/ reprintsandpermissions/

Journal peer review information: Nature Communications thanks Masaru Kuno and the other anonymous reviewer(s) for their contribution to the peer review of this work.

Publisher's note: Springer Nature remains neutral with regard to jurisdictional claims in published maps and institutional affiliations.

cc (i) Open Access This article is licensed under a Creative Commons Attribution 4.0 International License, which permits use, sharing, adaptation, distribution and reproduction in any medium or format, as long as you give appropriate credit to the original author(s) and the source, provide a link to the Creative Commons license, and indicate if changes were made. The images or other third party material in this article are included in the article's Creative Commons license, unless indicated otherwise in a credit line to the material. If material is not included in the article's Creative Commons license and your intended use is not permitted by statutory regulation or exceeds the permitted use, you will need to obtain permission directly from the copyright holder. To view a copy of this license, visit http://creativecommons.org/ licenses/by/4.0/.

(C) The Author(s) 2019 